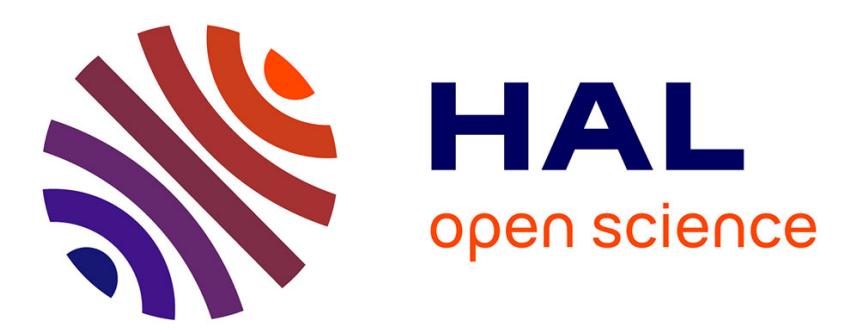

\title{
Caractérisation électronique d'interfaces profondes Al-InP
}

\author{
C. Barret, F. Vergand, H. Maaref, C. Sénémaud, C. Bonnelle
}

\section{To cite this version:}

C. Barret, F. Vergand, H. Maaref, C. Sénémaud, C. Bonnelle. Caractérisation électronique d'interfaces profondes Al-InP. Revue de Physique Appliquée, 1989, 24 (4), pp.439-446. 10.1051/rphysap:01989002404043900 . jpa-00246065

\section{HAL Id: jpa-00246065 https://hal.science/jpa-00246065}

Submitted on 1 Jan 1989

HAL is a multi-disciplinary open access archive for the deposit and dissemination of scientific research documents, whether they are published or not. The documents may come from teaching and research institutions in France or abroad, or from public or private research centers.
L'archive ouverte pluridisciplinaire HAL, est destinée au dépôt et à la diffusion de documents scientifiques de niveau recherche, publiés ou non, émanant des établissements d'enseignement et de recherche français ou étrangers, des laboratoires publics ou privés. 


\title{
Caractérisation électronique d'interfaces profondes Al-InP
}

\author{
C. Barret ( $\left.{ }^{(}\right)$, F. Vergand ( $\left.{ }^{2}\right)$, H. Maaref $\left({ }^{(1,2}\right)$, C. Sénémaud $\left({ }^{2}\right)$ et C. Bonnelle $\left(^{2}\right)$ \\ (1) Institut d'Electronique Fondamentale, UA22-CNRS, Université Paris Sud, Bâtiment 220, 91405 Orsay \\ Cedex, France \\ $\left.{ }^{2}\right)$ Laboratoire de Chimie Physique UA176-CNRS, Université Pierre et Marie Curie, 11 rue Pierre et Marie \\ Curie, 75231 Paris Cedex 05, France
}

(Reçu le $1^{\text {er }}$ juillet 1988, révisé le 18 octobre 1988, accepté le 12 janvier 1989)

\begin{abstract}
Résumé. - Des interfaces Al-InP (110) et (100) préparées par différentes méthodes ont été caractérisées par $I-V, C-V$, SCS, EXES. Ces mesures confirment l'existence sur InP n d'une hauteur de barrière significative $(>0,35 \mathrm{eV})$ et sensible à la méthode de préparation. Elles mettent en évidence la présence d'états d'interface discrets pour les échantillons clivés, ou répartis dans un continuum pour ceux ayant subi un nettoyage chimique. Des modifications très nettes de la densité des états $\mathrm{p}$ du phosphore sont observées aux différentes interfaces par rapport à InP en volume. Ceci implique la présence d'une zone interfaciale perturbée sur plusieurs dizaines d'angströms.

Abstract. - Al-InP (110) and (100) interfaces, obtained by means of various methods have been characterized by $I-V, C-V$, SCS, EXES. The measurements confirm the existence, for the n-InP material, of a significant barrier height $(>0.35 \mathrm{eV})$ which is sensitive to the method of preparation. They show the presence of discrete interface states for cleaved samples and of states lying in a continuum for chemically etched samples. Very clear modifications, compared to the bulk, of the $p$ density of states of phosphorus are observed at the various interfaces. They imply that the perturbed interfacial region is several tens of $\AA$ thick.
\end{abstract}

\section{Introduction.}

$\mathrm{Au}$ cours des dernières années, la connaissance sur les interfaces métal-semi-conducteur (M-S) a considérablement évolué. De nombreuses méthodes d'analyse des surfaces (Auger, LEED, Photoémission $\mathrm{X}$ ou $\mathrm{U}-\mathrm{V} . .$.$) ont été appliquées à des semi-$ conducteurs recouverts progressivement par des dépôts métalliques de l'ordre de la couche monoatomique. Ces études ont démontré, particulièrement dans le cas des semi-conducteurs III-V, la complexité de la structure de la plupart des interfaces M-S. La mise en évidence de phénomènes tels que la reconstruction des surfaces, l'interdiffusion des espèces atomiques, la création de nouvelles liaisons chimiques, etc... a conduit à remettre en cause l'existence d'interfaces abruptes à l'échelle atomique et à suggérer la présence d'une zone interfaciale de structure physico-chimique plus ou moins complexe séparant le métal et le semi-conducteur «parfait » [1]. Il ne fait aucun doute que les caractéristiques de cette zone interfaciale influent profondément sur les pro- priétés électriques du contact M-S. De nombreux modèles ont été proposés, faisant intervenir les notions de réactivité chimique du métal [2], de travail de sortie effectif [3] et surtout celle d'états d'interfaces et provenant de défauts ponctuels $[4,5]$ ou de liaisons déformées [6].

Cependant, à l'heure actuelle, aucun de ces modèles ne peut prétendre à lui seul expliquer de façon complète le comportement électrique des interfaces M-S. Pour progresser il est nécessaire de pouvoir coupler les caractérisations électriques et les analyses physico-chimiques de la zone interfaciale. Or les premières ne sont possibles que pour des interfaces M-S «profondes » de type diode Schottky, où les épaisseurs des couches métalliques sont de plusieurs centaines à plusieurs milliers d'angströms. Par contre les méthodes de physique des surfaces rappelées ci-dessus ne peuvent s'appliquer qu'à des interfaces «natives » où le dépôt métallique n'excède pas quelques angströms.

Nous avons réalisé une étude des interfaces $\mathrm{Al}$ InP « profondes » en associant trois types de caracté- 
risation : mesure de barrière par méthode électrique $(I-V, C-V)$; caractérisation des états d'interface dans la bande interdite du semi-conducteur par spectroscopie de capacité Schottky (SCS) ; analyse des états de valence de l'aluminium et du phosphoré dans la zone interfaciale par spectroscopie d'émission $X$ induite par électron (EXES). Cette dernière méthode présente l'avantage de pouvoir étudier une zone d'une centaine d'angströms, elle-même enterrée sous une couche métallique de quelques centaines d'angströms. Un autre avantage est que les techniques SCS et EXES sont utilisées ex-situ et sont totalement indépendantes de la préparation des échantillons. On peut donc les appliquer à des interfaces réalisées par n'importe quelle technologie. Les résultats présentés ici portent sur des diodes obtenues soit par clivage, sous ultra-vide ou à l'air, soit par nettoyage chimique suivi ou non d'un bombardement ionique de très basse énergie (inférieure à $50 \mathrm{eV}$ ).

\section{Préparation des échantillons.}

Nous avons utilisé des plaquettes polies (100) de type $\mathrm{n}\left(N_{\mathrm{D}}=10^{15} \mathrm{~cm}^{-3}\right)$ ou de type $\mathrm{p}\left(N_{\mathrm{A}}=\right.$ $\left.5 \times 10^{17} \mathrm{~cm}^{-3}\right)$ pour les échantillons nettoyés chimiquement et des barreaux (110) de $4 \mathrm{~mm} \times 5 \mathrm{~mm}$ de type $\mathrm{n}\left(N_{\mathrm{D}}=1,5 \times 10^{16} \mathrm{~cm}^{-3}\right)$ et de type $\mathrm{p}\left(N_{\mathrm{A}}=\right.$ $\left.3,5 \times 10^{17} \mathrm{~cm}^{-3}\right)$ pour les échantillons clivés. Un contact ohmique est tout d'abord réalisé par dépôt et recuit d'alliage Au-Ge (type n) ou Au-Zn (type p). Les diodes Al-InP sont obtenues en déposant par effet Joule une couche d'aluminium, soit d'environ $100 \AA$ pour l'analyse par EXES, soit de 1000 à $2000 \AA$ pour l'étude par SCS ; le dépôt est réalisé en quelques dizaines de secondes à quelques minutes à partir d'un filament de tungstène chargé d'aluminium.

Le clivage sous ultra-vide de InP est réalisé après le début de l'évaporation d'aluminium dans un vide stabilisé à quelques $10^{-9}$ torr. Le clivage à l'air est effectué dans l'atmosphère du laboratoire puis le barreau est immédiatement placé dans l'enceinte ultra-vide. Le nettoyage chimique, après dégraissage (trichloréthylène, alcool méthylique, acétone) est réalisé par immersion de la plaquette pendant $2 \mathrm{~min}$ dans une solution d'acide fluorhydrique à $50 \%$ et pendant 1 min dans une solution d'acide chlorhydrique à $10 \%$. Le nettoyage est terminé par un rinçage à l'eau désionisée et un séchage dans l'azote sec. Le bombardement ionique $\left(\mathrm{Ar}^{+} E \leqslant 50 \mathrm{eV}\right)$ est réalisé à l'aide d'une source VEECO ICS 3 montée sur un banc à vide de pression de base $10^{-7}$ torr. La pression d'argon au cours du bombardement est de $3 \times 10^{-4}$ torr. La durée du bombardement est fixée à $60 \mathrm{~s}$ sous une densité de courant de $0,5 \mathrm{~mA} / \mathrm{cm}^{2}$. Pour limiter les risques de pollution de la surface après décapage, le début de la métallisation est effectué environ $15 \mathrm{~s}$ après l'arrêt du bombardement. Des diodes individuelles sont délimitées par photolithographie. Certaines subissent un traitement thermique dans un four à tube de quartz jusqu'à $300^{\circ} \mathrm{C}$ pendant $10 \mathrm{~min}$.

\section{Hauteur de barrière.}

La hauteur de barrière d'un contact M-S peut être obtenue à partir de la caractéristique courant-tension de la diode. En effet, si le mécanisme de transport prépondérant est l'effet thermoionique audessus de la barrière de Schottky on a :

$$
\begin{aligned}
I=S A^{*} T^{2} \exp & \left(-\phi_{\mathrm{BJ}} / k T\right) \times \\
& \times\left(\exp \left(q\left(V-r_{\mathrm{s}} I\right) / n k T\right)-1\right)
\end{aligned}
$$

où $S$ est l'aire de la diode, $A^{*}$ la constante de Richardson modifiée en tenant compte des masses effectives des porteurs $\left(9,4 \mathrm{~A} \mathrm{~cm}^{-2} \mathrm{~K}^{-2}\right.$ pour les électrons et $84 \mathrm{~A} \mathrm{~cm}^{-2} \mathrm{~K}^{-2}$ pour les trous dans InP), $T$ la température absolue, $q$ la charge de l'électron et $k$ la constante de Boltzmann. Une courbe $I-V$ est conditionnée par trois paramètres : la hauteur de barrière $\phi_{\mathrm{BJ}}$, la résistance série $r_{\mathrm{S}}$ et le coefficient d'idéalité $n$. Ces trois paramètres sont extraits par une régression linéaire par moindres carrés. Les courbes expérimentales relatives à InP de type $n$ et InP de type $\mathrm{p}$ sont représentées sur la figure 1 pour les différents types de préparation utilisés. Les valeurs de $\phi_{\mathrm{BJ}}$ et $n$ sont regroupées dans le tableau I.

Une autre méthode pour obtenir la hauteur de barrière consiste à tracer $C^{-2}$ en fonction de $V, C$

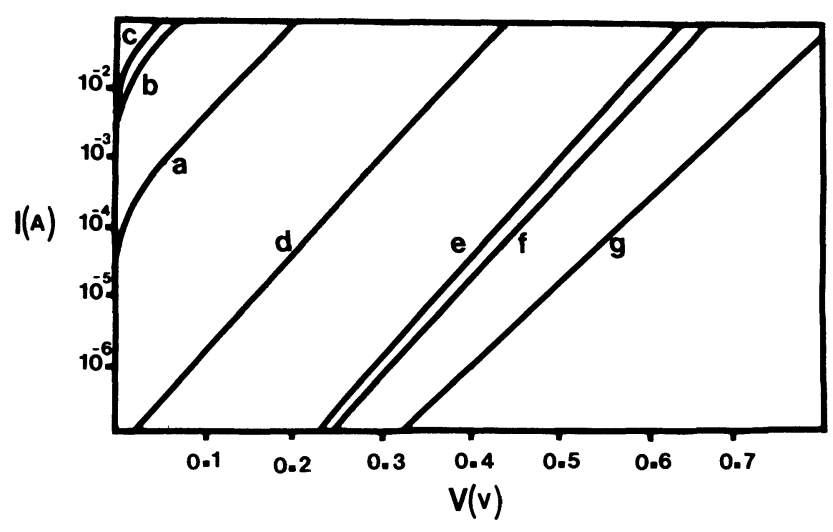

Fig. 1. - Caractéristiques courant-tension de diodes AlInP à température ambiante. Type $\mathrm{n}$ : (a) nettoyage chimique, (b) clivage sous ultra-vide, (c) nettoyage chimique + bombardement ionique. Type $p:$ (d) nettoyage chimique, (e) nettoyage chimique + bombardement ionique, (f) clivage sous ultra-vide, (g) clivage à l'air.

[I-V curves at $300 \mathrm{~K}$ for Al-InP. $\mathrm{n}$ type : (a) chemically etched, (b) UHV cleaved, (c) chemically etched + ion bombardment. p type : (d) chemically etched, (e) chemically etched + ion bombardment, (f) UHV cleaved, (g) air cleaved.] 
Tableau I. - Caractérisation électrique des diodes Schottky.

[Electrical characterization of Schottky diodes.]

\begin{tabular}{|c|c|c|c|c|c|}
\hline \multirow{2}{*}{$\begin{array}{l}\text { Préparation de la } \\
\text { surface d'InP }\end{array}$} & \multirow{2}{*}{$\begin{array}{l}\text { (110) clivée } \\
\text { sous ultra-vide }\end{array}$} & \multirow{2}{*}{$\begin{array}{l}\text { (110) clivée } \\
\text { à l'air }\end{array}$} & \multicolumn{3}{|c|}{ (100) nettoyée chimiquement } \\
\hline & & & $\begin{array}{l}\text { nettoy. chim. } \\
\text { seul }\end{array}$ & $\begin{array}{c}+ \text { bombardement } \\
\mathrm{Ar}^{+} E \leqslant 50 \mathrm{eV}\end{array}$ & $\begin{array}{l}+\mathrm{Ar}^{+} \\
+ \text {recuit } \\
300^{\circ} \mathrm{C}\end{array}$ \\
\hline Type $\mathrm{n}\left\{\begin{array}{l}\phi_{\mathrm{BJ}}(\mathrm{eV}) \\
n\end{array}\right.$ & $\begin{array}{l}0,37 \\
1,09\end{array}$ & $\begin{array}{l}\text { (a) } \\
\text { (a) }\end{array}$ & $\begin{array}{l}0,49 \\
1,16\end{array}$ & $\begin{array}{l}0,37 \\
1,05\end{array}$ & $\begin{array}{l}0,47 \\
1,04\end{array}$ \\
\hline Type $\mathrm{p}\left\{\begin{array}{l}\phi_{\mathrm{BJ}}(\mathrm{eV}) \\
n\end{array}\right.$ & $\begin{array}{l}0,94 \\
1,17\end{array}$ & $\begin{array}{l}0,95 \\
1,3\end{array}$ & $\begin{array}{l}0,75 \\
1,2\end{array}$ & $\begin{array}{l}0,92 \\
1,15\end{array}$ & $\begin{array}{l}0,79 \\
1,18\end{array}$ \\
\hline
\end{tabular}

(a) non reproductible.

étant la capacité différentielle mesurée en petit signal $(10 \mathrm{mV})$ et haute fréquence $(100 \mathrm{kHz})$ et $V$ la polarisation statique. Si la distribution de charge électrique dans la zone de charge d'espace est uniforme, la courbure de bandes est parabolique et $C^{-2}-V$ est une droite. De son abscisse à l'origine $V_{0}$ on déduit la hauteur de barrière $\phi_{\mathrm{BC}}$ par

$$
\phi_{\mathrm{BC}}=q V_{0}+\xi+k T
$$

$\xi$ étant la distance séparant en volume le niveau de Fermi du semi-conducteur de la bande de conduction (type $n$ ) ou de valence (type $p$ ). Pour les échantillons clivés sous ultra-vide les caractéristiques $C^{-2}-V$ sont parfaites et les valeurs obtenues par $\phi_{\mathrm{BC}}$ sont $0,39 \mathrm{eV}$ pour le type $\mathrm{n}$ et $0,98 \mathrm{eV}$ pour le type $\mathrm{p}$. Par contre, lorsque la surface d'InP s'est trouvée au contact de l'air avant métallisation (clivage à l'air, nettoyage chimique), les courbes $C^{-2}-V$ présentent des non-linéarités plus ou moins fortes et sont inexploitables pour obtenir une valeur précise de $\phi_{\mathrm{BC}}$.

Malgré un assez grand nombre d'études, il existe toujours une forte controverse sur la valeur de la barrière de Schottky à l'interface Al-InP. Le cas le plus typique est celui du contact Al-InP n clivé sous ultra-vide pour lequel, la reproductibilité des conditions expérimentales devrait pourtant être la meilleure. Deux types de résultats ont été publiés qui indiquent soit un comportement ohmique $[7,8]$, soit une barrière de l'ordre de $0,3 \mathrm{eV}[9,10]$. Notre étude confirme l'existence d'une barrière relativement importante (de l'ordre de $0,4 \mathrm{eV}$ ) et va à l'encontre de la classification des hauteurs de barrière en fonction de la réactivité du métal proposé par Brillson [1, 2] et Williams [11].

Lorsque la surface clivée d'InP séjourne quelques minutes à l'air avant la métallisation nous obtenons, sur les échantillons de type $\mathrm{n}$, des résultats non reproductibles; la barrière apparente est inférieure ou égale à celle obtenue par clivage sous ultra-vide. Cela est à rapprocher des résultats de Williams [7, 12] qui observe un comportement ohmique et de Newman [10] qui mesure une barrière de $0,34 \mathrm{eV}$. Une explication possible est que l'interface Al-ImP clivée à l'air présente des inhomogénéités de barrière dues aux réactions entre l'aluminium, l'oxygène et le semi-conducteur. Il suffit que l'aire où se produit une faible barrière (par exemple liée à l'existence d'oxyde d'indium $\operatorname{In}_{2} \mathrm{O}_{3}$ [7]) varie légèrement d'un échantillon à l'autre pour expliquer la dispersion observée. Il est intéressant de remarquer que sur le matériau de type $\mathrm{p}$ les caractéristiques $I-V$ et $C-V$, bien que non idéales, sont beaucoup plus reproductibles. En effet les zones à faible barrière existant sur le type $\mathrm{n}$ deviennent des zones à forte barrière sur le type $p$ et n'influencent pratiquement pas le comportement électrique de la diode (13).

Dans le cas du nettoyage chimique la présence d'une couche interfaciale d'épaisseur significative provoque une valeur importante du coefficient d'idéalité $n$. Dans ce cas les valeurs de hauteur de barrière indiquées dans le tableau I sont des valeurs minimales. Par comparaison des résultats obtenus sur le matériau $n$ et sur le matériau $p$ on peut estimer que $\phi_{\mathrm{Bn}}$ est de l'ordre de 0,5 à $0,55 \mathrm{eV}$, en accord avec plusieurs autres valeurs publiées [12, 14].

Lorsque le nettoyage chimique est suivi d'un bombardement ionique de très faible énergie, on obtient un coefficient d'idéalité proche de l'unité. Ceci prouve l'efficacité de ce traitement pour éliminer la couche d'oxyde natif sans créer de défauts de structure catastrophiques, comme cela se produit en utilisant des ions de plus fortes énergies [15, 17]. Par ailleurs le bombardement ionique provoque une diminution de $\phi_{\mathrm{B}}$ sur le matériau de type $\mathrm{n}$ et une augmentation sur le matériau de type p. Ceci est conforme aux nombreuses observations similaires 
effectuées sur des interfaces M-Si $[18,19]$ et MGaAs [20-22] bombardées. Si ces échantillons subissent un traitement thermique modéré $\left(300^{\circ} \mathrm{C}\right)$, la hauteur de barrière décroît pour le type $\mathrm{p}$ et croît pour le type $\mathrm{n}$ revenant à une valeur comparable à celle obtenue sur des surfaces non bombardées. Des résultats similaires ont été observés pour des interfaces Al-GaAs [22].

\section{Etats d'interface.}

Pour détecter un état d'interface par SCS, (cf. Réfs. [23-25]), il est nécessaire de modifier sa charge. Ceci est possible électriquement en agissant sur les probabilités d'échanges entre l'état d'interface et les bandes du semi-conducteur ou du métal. Lorsqu'on polarise une diode Schottky en direct, on déplace dans la bande interdite le quasi-niveau de Fermi des porteurs majoritaires [26]. Ceci revient à modifier la concentration des porteurs libres à l'interface et donc la probabilité de capture d'un de ces porteurs par l'état d'interface. Ainsi si on superpose un petit signal alternatif à une polarisation directe continue, on obtient une capacité $C_{\text {is }}$ due à la modulation de charge de l'état qui s'ajoute à la capacité $C_{\text {sc }}$ due à la variation de charge en limite de zone de charge d'espace. $C_{\text {is }}$ dépend de la fréquence, de la température et de la polarisation par l'intermédiaire du temps de relaxation des états et de leurs fonctions d'occupation.

Plusieurs méthodes expérimentales ont été développées sur ce principe, elles utilisent pour effectuer un balayage en énergie à l'intérieur de la bande interdite du semi-conducteur, soit simplement une modification de la polarisation directe à température constante $[27,28]$, soit un balayage lié en polarisation et température qui maintient la conductance dynamique de la diode constante [29]. C'est cette technique que nous avons appliquée ici aux interfaces Al-InP.

Dans ce mode de fonctionnement, à un état d'énergie $E_{\mathrm{s}}$ correspond un pic $C_{\text {is }}$ à une température $T_{\mathrm{p}}$ telle que :

$$
\begin{aligned}
\left(k T_{\mathrm{p}}\right)^{-1}= & \left(E_{\mathrm{c}}-E_{\mathrm{s}}\right)^{-1} \times \\
& \times \operatorname{Ln}\left[\left(c_{\mathrm{n}} N_{\mathrm{c}}\right)\left(4 \pi^{2} f^{2}+c_{\mathrm{n}}^{2} n_{\mathrm{s}}^{2}\right)^{-\frac{1}{2}}\right]
\end{aligned}
$$

où $N_{\mathrm{c}}$ est la densité d'états équivalente dans la bande de conduction, $n_{\mathrm{s}}$ la densité des électrons libres à la surface du semi-conducteur, $c_{n}$ le coefficient de capture de ces électrons par l'état d'interface et $f$ la fréquence du signal sinusoïdal.

Cette relation est valable pour une interface $\mathrm{M}-\mathrm{S}$ de type $n$. On obtient immédiatement par transposition une relation équivalente pour une interface $M-S$ de type $\mathrm{p}$ en faisant intervenir la distance entre l'état d'interface et le sommet de la bande de valence.
Pour pouvoir explorer la majeure partie de la bande interdite, il est nécessaire que les barrières de Schottky $\phi_{\mathrm{Bn}}$ et $\phi_{\mathrm{Bp}}$ soient voisines de $E_{\mathrm{c}} / 2$ [23]. Ceci n'est malheureusement pas le cas pour nos échantillons. La faible valeur de $\phi_{\mathrm{Bn}}$ rend impossible toute étude entre le niveau de Fermi et la bande de conduction. Nous ne présentons donc que les résultats obtenus sur des interfaces de type $\mathrm{p}$; la zone explorée s'étend du milieu de la bande interdite jusqu'à $E_{\mathrm{V}}+0,1 \mathrm{eV}$ pour un balayage en température allant de la température ambiante à celle de l'azote liquide.

La variation avec la température de la capacité $C_{\text {is }}$ associée à la réponse des états d'interface est reportée sur la figure 2 à différentes fréquences pour des interfaces clivées sous vide et à l'air. Dans les deux cas on observe un pic dont le maximum se déplace vers les basses températures lorsque la fréquence diminue, conformément à l'équation (3). Cette équation peut être exploitée pour des fréquences supérieures à une fréquence critique $f_{\mathrm{c}}=$ $c_{\mathrm{n}} N_{\mathrm{c}} / 2 \pi$ en traçant $\left(k T_{\mathrm{p}}\right)^{-1}$ en fonction de Ln $\left(f \cdot\left(T_{\mathrm{A}} / T_{\mathrm{p}}\right)^{2}\right) . T_{\mathrm{A}}$ est la température ambiante et le terme en $\left(T_{\mathrm{A}} / T_{\mathrm{p}}\right)^{2}$ provient de la dépendance en température de $c_{\mathrm{n}} N_{\mathrm{c}}$. On obtient une droite dont la pente donne l'énergie $E_{\mathrm{s}}$ de l'état et l'abscisse à l'origine le coefficient de capture (ou la section efficace de capture) des porteurs majoritaires. Cette

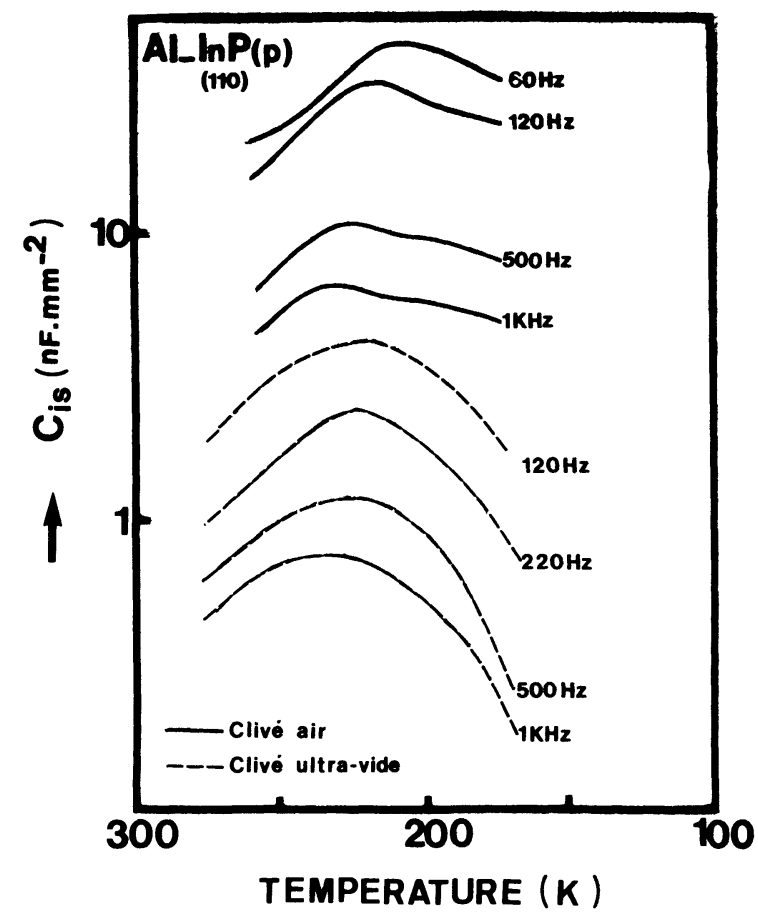

Fig. 2. - Variation avec la température de la capacité due à la réponse des états d'interface pour $\mathrm{Al}$-InP $\mathrm{p}$ clivé sous ultra-vide et à l'air.

[Variation in interface states capacitance as a function of temperature for UHV cleaved and air cleaved Al-InP p interfaces at various frequencies.] 


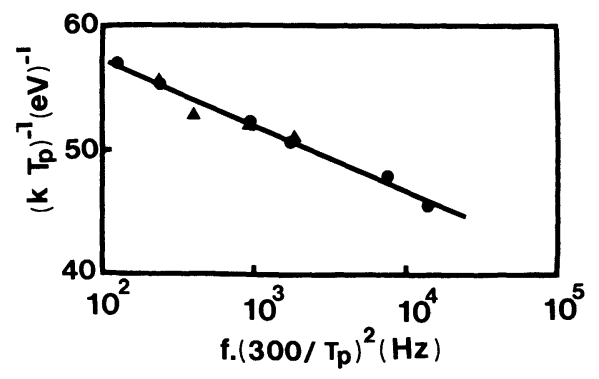

Fig. 3. - Signature de l'état localisé sur la figure 2: $(\bullet)$ clivage à l'air, ( $\Delta$ ) clivage sous ultra-vide. La droite obtenue correspond à un état situé à $0,4 \mathrm{eV}$ au-dessus du sommet de la bande de valence et ayant une section efficace de capture de l'ordre de $10^{-13} \mathrm{~cm}^{2}$.

[Signature of the localised state observed in figure 2 : $(\bullet)$ air cleaved, ( $\Delta$ ) UHV cleaved. The curve corresponds to a state located at $E_{\mathrm{V}}+0.4 \mathrm{eV}$ with a hole capture cross-section around $10^{-13} \mathrm{~cm}^{2}$.]

droite est une signature de l'état car elle ne dépend que de ses caractéristiques et non de celles de la diode [29]. Le résultat reporté sur la figure 3 indique la présence d'un état identique pour les deux interfaces. Cet état est situé à $0,4 \mathrm{eV}$ au-dessus du sommet de la bande de valence et a une section efficace de

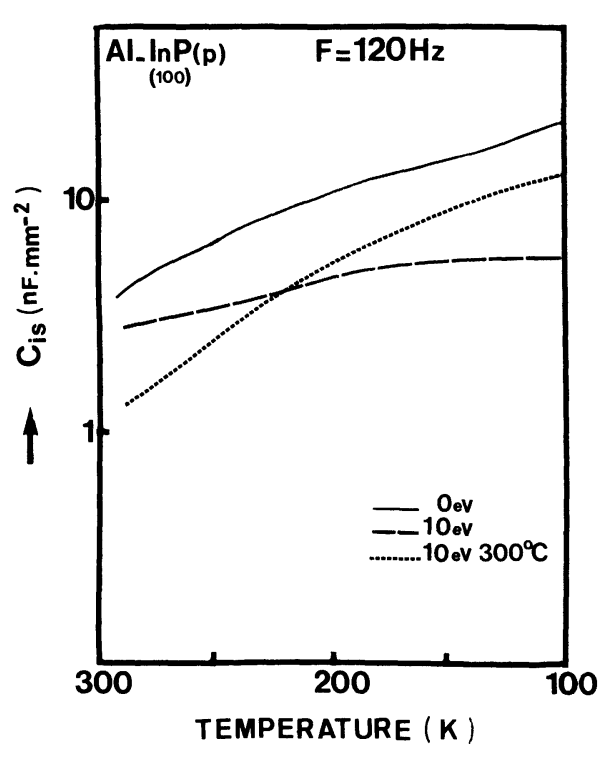

Fig. 4. - Variation avec la température de la capacité due à la réponse des états d'interface à une fréquence de $120 \mathrm{~Hz}$ pour différentes interfaces Al-InP p (100): $(-)$ nettoyage chimique, $(---)$ nettoyage chimique plus bombardement ionique, $(. . .$.$) nettoyage$ chimique plus bombardement ionique plus recuit à $300^{\circ} \mathrm{C}$.

[Variation in interface states capacitance as a function of temperature at $\mathrm{f}=120 \mathrm{~Hz}$ for various Al-InP p (100) interfaces : $(\longrightarrow)$ chemically etched, $(--\longrightarrow)$ chemically etched + ion bombardment, $(. . . .$.$) chemically$ etched + ion bombardment + thermal annealing $300^{\circ} \mathrm{C}$.] capture des trous de l'ordre de $10^{-13} \mathrm{~cm}^{2}$. On constate par ailleurs sur la figure 2 , que les valeurs de $C_{\text {is }}$ sont plus élevées d'environ une décade pour les surfaces clivées à l'air. Cela peut signifier que la densité de l'état $E_{\mathrm{V}}+0,4 \mathrm{eV}$ est plus élevée. Mais l'étalement de la partie droite des courbes, dans le cas du clivage à l'air, montre que l'état à $E_{\mathrm{V}}+$ $0,4 \mathrm{eV}$ n'est pas le seul à contribuer à $C_{\text {is }}$. Comme cela a été observé sur GaAs [25], il semble que des états supplémentaires énergétiquement peu localisés (continuum ou bande large) apparaissent lorsque la surface d'InP a séjourné à l'air.

Sur la figure 4 sont reportées trois courbes $C_{\text {is }}(T)$ obtenues à la même fréquence $(120 \mathrm{~Hz})$ pour des interfaces $\mathrm{Al} / \mathrm{InP}$ (100) préparées respectivement par nettoyage chimique simple; nettoyage chimique et bombardement $\mathrm{Ar}^{+}$; nettoyage chimique, bombardement $\mathrm{Ar}^{+}$et recuit $\left(300^{\circ} \mathrm{C}\right)$. On constate l'absence de structure. Il n'y a en particulier aucun pic à $220 \mathrm{~K}$ qui pourrait correspondre à ce qui est observé pour les interfaces clivées. Ceci indique qu'il n'y a pas d'état énergétiquement localisé (tout au moins dans la zone de la bande interdite explorée) ou que leur densité est négligeable par rapport à celle du continuum observé. Ce continuum crô̂t vers la bande de valence et a un profil et une densité qui dépendent de la préparation de l'échantillon.

\section{Analyse des états de valence.}

La distribution des états de valence $3 p$ du phosphore dans InP est obtenue à partir de l'émission $K \boldsymbol{\beta}$; celle-ci correspond à la décroissance radiative d'une lacune 1s par transition électronique $3 p \rightarrow 1 s$.

Lors de l'étude de l'interface par EXES [30], l'ionisation $1 \mathrm{~s}$ du phosphore est produite à l'aide d'électrons dont l'énergie cinétique maximale est, après traversée de l'aluminium, supérieure de $300 \mathrm{eV}$ à l'énergie seuil d'ionisation (2 143,5 eV); l'énergie $E_{0}$ du faisceau d'électrons incidents est déterminée pour chaque échantillon à l'aide d'une courbe d'excitation. Dans ces conditions, l'épaisseur d'InP analysée est de l'ordre de $100 \AA$. Les spectres des monocristaux en volume sont obtenus avec une énergie des électrons incidents égale à $4000 \mathrm{eV}$.

Les spectres ont été réalisés à l'aide de spectromètres à cristal courbé fonctionnant sous vide par réflexion ; la résolution en énergie est de l'ordre de $0,2 \mathrm{eV}$ dans le domaine étudié.

L'émission $\mathrm{K} \beta(3 \mathrm{p} \rightarrow 1 \mathrm{~s})$ de InP à l'interface $\mathrm{Al}$ InP p (110) clivé sous ultra-vide est présentée figure 5a comparativement à celle de InP $\mathrm{p}$ analysé en volume. La distribution de l'intensité émise est portée en fonction de l'énergie des photons; les énergies de liaison des électrons de valence sont données par $E-E_{\mathrm{V}}$, le sommet $E_{\mathrm{V}}$ de la bande de valence étant positionné au point d'inflexion de la bande d'émission. 


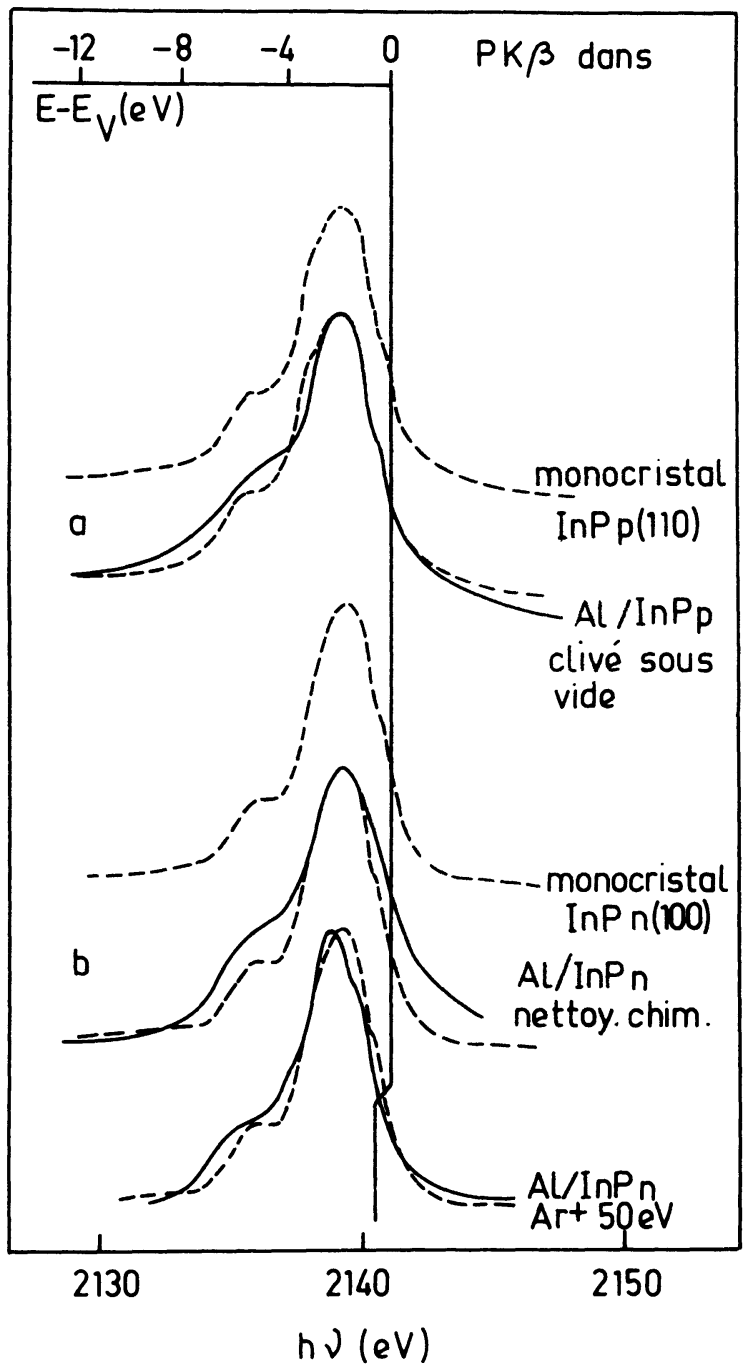

Fig. 5. - Bande d'émission $K \boldsymbol{\beta}$ du phosphore (a) à l'interface Al-InP p (110) clivée sous ultra-vide comparée à la bande d'émission du monocristal d'InP $\mathrm{p}$ en volume, (b) aux interfaces Al-InP n (100), nettoyées chimiquement et bombardées par des ions $\mathrm{Ar}^{+}$, comparées à la bande d'émission du monocristal d'InP $\mathrm{n}$ en volume.

[P K $\beta$ emission (a) at the UHV cleaved interface Al-p-InP (110) compared to the emission band of bulk p-InP, (b) at the chemically etched and $\mathrm{Ar}^{+}$bombarded interfaces $\mathrm{Al}$ $\mathrm{n}$-InP (100) compared to the emission band of bulk n-InP.]

La forme de la distribution des états $\mathrm{p}$ du phosphore à l'interface diffère notablement de celle observée pour le matériau en volume : le pic principal apparaît contracté, cette contraction étant due essentiellement à la disparition de l'épaulement à $E_{\mathrm{V}}-2,6 \mathrm{eV}$. La structure située vers les grandes énergies de liaison s'élargit et la densité d'états est renforcée au voisinage de $E_{\mathrm{V}}-5 \mathrm{eV}$ et au-delà de $E_{\mathrm{V}}-6 \mathrm{eV}$, c'est-à-dire dans un domaine d'énergie qui correspond à des états hybridés sp. Les maximums des deux émissions sont observés à la même énergie. Les niveaux $1 s$ et $3 p$ qui interviennent dans ces transitions sont en effet également déplacés par la courbure de bande.

L'émission $\mathrm{K} \beta$ de l'interface Al-InP n (100) nettoyée chimiquement est présentée figure $5 b$ comparativement à celle de InP $n$ en volume. Cette distribution est très élargie et moins structurée que celle du composé massif. Il apparaît là encore un renforcement des états $\mathrm{p}$ du côté des grandes énergies de liaison mais ce renforcement existe aussi en haut de la bande. Les maximums des deux émissions coïncident à la précision expérimentale. L'élargissement et la perte de structures pourraient être expliqués par une superposition de distributions de formes légèrement différentes due à l'inhomogénéité de la zone interfaciale.

Pour ce type d'échantillon, la surface de InP est oxydée avant métallisation. Cette couche d'oxyde pourrait être réduite par l'aluminium [12]. Ceci entraînerait la formation de $\mathrm{Al}_{2} \mathrm{O}_{3}$ à l'interface. Afin de rechercher la présence éventuelle de cet oxyde, nous avons analysé l'émission $K \boldsymbol{\beta}(3 \mathrm{p} \rightarrow 1 \mathrm{~s})$ de la couche d'aluminium. Le spectre est présenté figure 6 comparativement à celui d'une couche d'aluminium

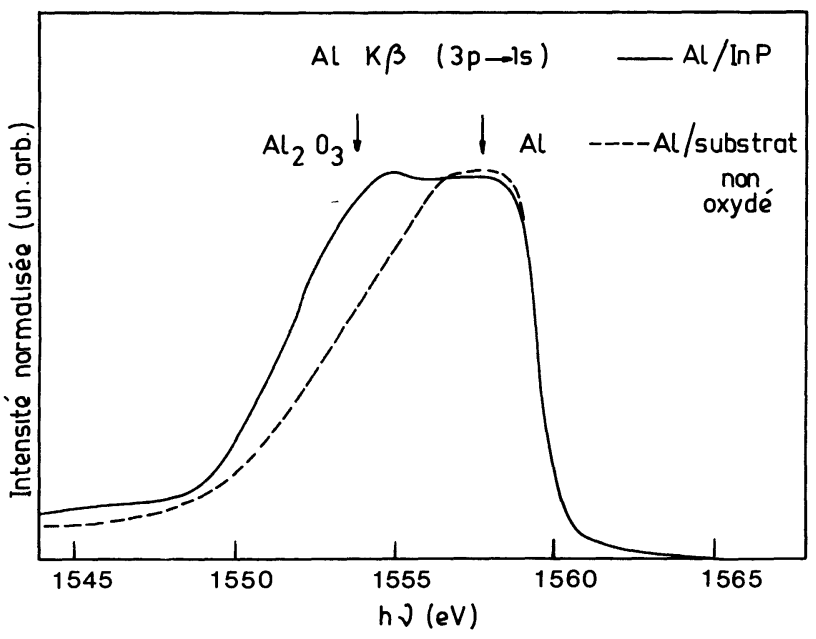

Fig. 6. - Bande d'émission $K \boldsymbol{\beta}$ de l'aluminium de l'échantillon Al-InP n nettoyé chimiquement comparée à celle de l'aluminium sur substrat non oxydé.

[Al K $\beta$ emission band of $\mathrm{Al}$-n-InP chemically etched sample compared to that of aluminium deposited on a non oxidized substrate.]

de même épaisseur, déposée dans les mêmes conditions sur un substrat exempt d'oxyde (une couche de nickel déposée sous vide juste avant l'évaporation de l'aluminium). Le spectre d'Al sur InP présente une proportion d'oxyde beaucoup plus importante que le spectre de $\mathrm{Al}$ sur $\mathrm{Ni}$. Dans ce dernier cas $\mathrm{Al}_{2} \mathrm{O}_{3}$ est formé uniquement sur la face externe de l'échantillon lors de son passage à l'air entre l'évaporateur et le spectromètre. La forte quantité d' $\mathrm{Al}_{2} \mathrm{O}_{3}$ présente dans l'échantillon $\mathrm{Al}$ sur InP suggère qu'il $\mathrm{y}$ a bien effectivement réduction de l'oxyde superficiel par l'aluminium. 
Lorsque InP subit un bombardement par des ions $\mathrm{Ar}^{+}$de $50 \mathrm{eV}$ avant métallisation, l'émission $\mathrm{K} \beta \mathrm{du}$ phosphore est fortement modifiée par rapport à celle obtenue sans bombardement (Fig. 5b) : le maximum se déplace de $-0,4 \mathrm{eV}$ et la largeur de la bande diminue, des structures étant nettement observables de part et d'autre du maximum. Si l'on ajuste le bord de la bande de valence avec celui de l'échantillon analysé en volume, les deux distributions diffèrent essentiellement par la disparition de la structure $E_{\mathrm{V}}-2,6 \mathrm{eV}$ à l'interface et par une modification de forme du haut de la bande de valence.

Il est possible d'interpréter les structures de l'émission $\mathrm{K} \boldsymbol{\beta}$ de InP monocristallin par des structures de la densité d'états. Toutefois la courbe théorique [31] correspond à la densité d'états totale tandis que la courbe observée correspond à la densité d'états partielle $\mathrm{p}$ du phosphore. Divers types de désordre peuvent renforcer la densité d'états entre les structures de grande énergie de liaison ainsi qu'en haut de la bande de valence [32]. Cela a été observé expérimentalement par exemple pour InP amorphe [33]. C'est donc à un effet de désordre que nous attribuons les modifications observées pour l'échantillon nettoyé chimiquement et pour l'échantillon bombardé. Pour l'échantillon clivé, qui ne présente pas de modification du haut de la bande de valence mais seulement un renforcement de la densité des états de grande énergie de liaison, le type de désordre serait différent du précédent. Nos résultats mettent donc en évidence dans tous les cas la présence d'une couche partiellement désordonnée sur plusieurs dizaines d'angströms à l'interface. Dans le cas de l'échantillon nettoyé chimiquement une couche d'oxyde pourrait rester présente ce qui expliquerait l'élargissement de la distribution.

Il reste à expliquer le déplacement de $\mathbf{K} \boldsymbol{\beta}$ observé pour l'interface Al-InP $\mathrm{n}$ nettoyé chimiquement et bombardé. D'après les résultats de Kendelewicz et al. [34], le niveau de cœur $2 p$ du phosphore pour InP $\mathrm{n}$ recouvert de quelques angströms d'aluminium est décalé vers les faibles énergies de liaisons par rapport à l'échantillon nu, tandis que le maximum principal de la bande de valence reste à la même énergie. Le niveau de cœur 1s doit subir un décalage analogue à celui du niveau $2 p$, ce qui expliquerait le déplacement que nous observons pour $\mathrm{K} \beta$. Ce déplacement pourrait être dû à la présence d'AlP. Cependant la proportion d'AlP que l'on attend pour une surface exempte de défauts comme une surface clivée [34] ou reconstruite [35, 36] serait trop faible pour donner lieu à un déplacement observable. Nos résultats semblent montrer que, dans le cas de l'échantillon bombardé, AIP serait présent sur plusieurs dizaines d'angströms, sa formation étant favorisée par les défauts créés sous bombardement ionique.

\section{Conclusion.}

Nous avons étudié des interfaces $\mathrm{Al}$-InP obtenues par diverses méthodes de préparation (clivage sous ultra-vide, clivage à l'air, nettoyage chimique suivi ou non d'un bombardement ionique). Nous avons appliqué à ces interfaces trois types de caractérisation. A l'aide des caractéristiques $I-V$ et $C-V$ nous avons déterminé la hauteur de la barrière de Schottky. Nous montrons que celle-ci est sensible à la méthode de préparation, passant de $0,35-0,40 \mathrm{eV}$ pour les surfaces clivées à $0,50-0,55 \mathrm{eV}$ pour les surfaces nettoyées chimiquement. Le résultat obtenu sur les surfaces clivées confirme la valeur indiquée par Newman [10]. La spectroscopie de Capacité Schottky met clairement en évidence une différence importante dans la distribution des états d'interface dans la moitié inférieure de la bande interdite : pour les interfaces (110) il apparaît un état énergétiquement localisé autour de $E_{\mathrm{V}}+0,4 \mathrm{eV}$; pour les interfaces (100) nettoyées chimiquement, on observe un continuum d'états croissant vers la bande de valence. La spectroscopie d'émission $\mathrm{X}$ induite par électrons est une méthode de caractérisation des interfaces qui est appliquée pour la première fois ici à des contacts M-S. Cette méthode présente l'intérêt majeur de ne pas nécessiter l'emploi de couches métalliques ultra-minces. Elle permet donc d'obtenir des informations physico-chimiques sur la structure de l'interface profonde entre un métal ayant effectivement des propriétés de matériau massif et un semi-conducteur. Par ailleurs l'épaisseur de la zone interfaciale analysée est typiquement de l'ordre de la centaine d'angströms. Dans le cas des interfaces AlInP, nous montrons que des modifications importantes de la distribution $\mathrm{p}$ du phosphore se produisent. Ces observations indiquent la présence à l'interface Al-InP d'une zone structurellement perturbée et prouvent que l'épaisseur de cette zone interfaciale est de plusieurs dizaines d'angströms. Des expériences complémentaires sont en cours pour préciser le type de désordre.

\section{Bibliographie}

[1] Voir par exemple l'article de BRILlson L. J., Surf. Sci. Rep. 2 (1982) $\mathrm{n}^{\circ} 2$.

[2] BRILlson L. J., J. Vac. Sci. Technol. 15 (1987) 1378.
[3] Freeouf J. L., Woodall J. M., Appl. Phys. Lett. 39 (1981) 727.

[4] Spicer W. E., Chye P. R., Su C. Y., Lindau J., J. Vac. Sci. Technol. 16 (1979) 1427. 
[5] Newman N., Spicer W. E., Kendelewicz T., Lindau J., J. Vac. Sci. Technol. B 4 (1986) 931.

[6] Hasegawa H., Sawada T., Thin Solid Films 103 (1983) 119.

[7] Williams R. H., VARMA R. R., MCKinley A., J. Phys. C 10 (1977) 4545.

[8] Ismail A., Palau J. M., Lassabatère L., Revue Phys. Appl. 19 (1984) 205.

[9] SlOWIK J. H., RICHTER H. W., BRILlSON L. J., J. Appl. Phys. 58 (1985) 3154.

[10] Newman N., Kendelewicz Y., Bowman L., SPICER W. E., Appl. Phys. Lett. 48 (1985) 1176.

[11] Williams R. H., MONTGOMERY V., VARMa R. R., J. Phys. C 11 (1978) 1989.

[12] Williams R. H., Mclean A. B., Evans D. A. and HerRenden-HARKer W. G., J. Vac. Sci. Technol. B 4 (1986) 966.

[13] Barret C., Lu G. N., Neffati T., Revue Phys. Appl. 22 (1987) 1485.

[14] Christou A., Anderson W. T., Solid State Electron. 22 (1979) 857.

[15] Williams R. S., Nelson R. J., Schlier A. R., Appl. Phys. Lett. 36 (1980) 827.

[16] Williams R. S., Solid State Commun. 41 (1982) 153.

[17] Dautremont-Smith W. C., Feldman L. C., Thin Solid Films 105 (1983) 187.

[18] Mullins F. H., Brunschweiler A., Solid State Electron. 19 (1976) 47.

[19] Fonash S. J., AshoK S., Jingh R., Appl. Phys. Lett. 39 (1981) 423, Thin Solid Films 90 (1982) 231.

[20] Wang Y. X., Holloway P. H., J. Vac. Sci. Technol. B 2 (1984) 613.

[21] Wu C. S., Scott D. M., Chen W. X., LAU S. S., J. Electrochemi. Soc. 132 (1985) 918.
[22] Neffati T., Barret C., Lu G. N., MaARef H., Revue Phys. Appl. ; Solid State Electron. (sous presse).

[23] Barret C., Chekir F., Vapaille A., J. Phys. C 16 (1983) 2421.

[24] Muret P., Deneuville A., J. Appl. Phys. 53 (1982) 6289.

[25] Chekir F., Barret C., Vapaille A., J. Appl. Phys. 54 (1983) 6474.

[26] Crowell C. R., Beguwala M., Solid State Electron. 14 (1971) 1149.

[27] Muret P., Thèse d'état (Grenoble) 1985.

[28] Evans H. L., Wu X., Yang E. S., Ho P. S., J. Appl. Phys. 60 (1986) 3611.

[29] Chekir F., Barret C., Appl. Phys. Lett. 45 (1984) 1212.

[30] Fargues D., Vergand F., Bonnelle C., Surf. Sci. 163 (1985) 489.

[31] Chelikowski J. R., Cohen M. L., Phys. Rev. B 14 (1976) 556.

[32] Joannopoulos J. D., Cohen M. L., Phys. Rev. B 10 (1974) 1545.

[33] Ouchene M., Senemaud C., Belin E., Georghiu A., TheYe M. L., J. Non-Cryst. Solids 59, 60 (1983) 625.

[34] Kendelewicz T., Petro W. G., Lindau I., Spicer W. E., Phys. Rev. B 30 (1984) 5800.

[35] Houzay F., Moison J. M., Bensoussan M., J.'Vac. Sci. Technol. B 3 (1985) 756.

[36] Houzay F., Bensoussan M., Barthe F., Surf. Sci. 168 (1986) 347. 